\title{
Preparation of Platinum Decorated Laser-Induced Graphene Flexible Electrode and Its Application for Clenbuterol Detection
}

\author{
Hongwei Tang 1,!, Ying Zhong ${ }^{2,5, !}$, Xiangrong Zeng ${ }^{2,5, !}$, Yu Sang ${ }^{2,3}$, Furui Lin ${ }^{2}$, \\ Yifu Zhu', Zhongping Chen ${ }^{4, *}$, Lanjiao Xu ${ }^{3}$, Zhong Huang ${ }^{2}$, Peicong Zhou ${ }^{5, *}$ \\ ${ }^{1}$ College of Computer and Information Engineering, Jiangxi Agricultural University, Nanchang \\ 330045, China \\ ${ }^{2}$ College of Chemistry and Materials, Jiangxi Agricultural University, Nanchang 330045, China \\ ${ }^{3}$ Jiangxi Province Key Laboratory of Animal Nutrition/Engineering Research Center of Feed \\ Development, Jiangxi Agricultural University, Nanchang 330045, China \\ ${ }^{4}$ Agricultural Technology Extension Center of Jiangxi Province, Nanchang 330046, China \\ ${ }^{5}$ College of Engineering, Jiangxi Agricultural University, Nanchang 330045, China \\ *E-mail: czpjxaas@163.com, pc.zhou@jxau.edu.cn
}

Received: 27 October 2021 / Accepted: 29 November 2021 / Published: 5 January 2022

\begin{abstract}
The traditional preparation method of graphene is complicated in operation and high-cost, which severely limits the further application of graphene. Hence, a new strategy for the rapid, large-scale, lowcost preparation of graphene can effectively solve the current problems. In this work, laser-induced graphene (LIG) was rapidly prepared in one step based on polyimide (PI) film as the substrate, by using laser direct writing technology, and prepare LIG into a flexible electrode. Besides that, LIG flexible electrode was modified the by chemical method, sodium borohydride was used as reducing agent to reduce chloroplatinic acid, and Pt metal nanoparticles were loaded on the surface of LIG electrode in situ to prepare Pt-LIG composite electrode material. Compared with LIG electrode, Pt-LIG electrode has high conductivity, larger surface area and better electrocatalytic ability. It is used as a new type of electrochemical sensing platform to perform electrochemical detection of clenbuterol (CLB), obtaining a wide detection linear range of $0.1-820.9 \mu \mathrm{M}$, and a lower limit of detection (LOD) of $0.072 \mu \mathrm{M}$. Moreover, the CLB in the actual sample of beef was detected at the same time, and an effective and acceptable recovery rate was obtained.
\end{abstract}

Keywords: Laser-induced graphene; Pt nanoparticles; Flexible electrode; Electrochemical sensor; Clenbuterol.

\section{$\underline{\text { FULL TEXT }}$}

(C) 2022 The Authors. Published by ESG (www.electrochemsci.org). This article is an open access article distributed under the terms and conditions of the Creative Commons Attribution license (http://creativecommons.org/licenses/by/4.0/). 